\title{
Connections on Jordan modules and particle physics
}

\section{Alessandro Carotenuto*t \\ SISSA \\ E-mail: acarotenesissa.it}

We review differential calculus on modules of Jordan algebras, focusing on the exceptional Jordan algebra $J_{3}^{8}$ that, as shown by Michel Dubois-Violette in "Exceptional quantum geometry and particle physics" might provide the opportune mathematical background to overcome the main problems of noncommutative formulation of Standard Model, namely the quark lepton-symmetry and the three generations of particle. This a joint work with M. Dubois-Violette and L. Dabrowski.

Corfu Summer Institute 2017 'School and Workshops on Elementary Particle Physics and Gravity' 2-28 September 2017

Corfu, Greece

\footnotetext{
* Speaker.

${ }^{\dagger}$ Based on a joint work with Ludwik Dabrowski and Michel Dubois-Violette
} 


\section{Introduction}

Noncommutative spectral geometry in the lenguage of Connes's spectral triples is the most widely acclaimed mathematical formalization for the Standard Model of particle physics so far (see e.g. [5], [7], [6],[4]). The main cause behind this success lies in the possibility to extrapolate most of Standard Model features (above all the existence of the Higgs boson) from a purely mathematical context, provided by the well celebrated reconstruction theorem for spectral triples.

That said, this model exhibits some flaws: from a mathematical point of view, the reconstruction theorem would prescribe to take as gauge group the unitaries inside the $C^{*}$ algebra $\mathbb{C} \otimes \mathbb{H} \otimes M_{3}(\mathbb{C})$. While this prescription would lead naturally to the $U(1) \times S U(2)$ gauge symmetry, the $S U(3)$ colour symmetry of standard model has to be selected by hand (this is referred in mathematical litterature as unimodularity condition). From a more physical point of view, spectral triples do not provide any justification neither to the existence of three generations of particles nor to the quark-lepton symmetry.

As we shall review in the following sections, in [8] the author shows how these two missing features could be obtained by allowing Jordan algebras to play the role of quantum observables in the Standard Model.

In the last section we shall present some recent development in the theory (which are given with full details in [3]), in particular we will show the explicit description of the theory of connections for modules over Jordan algebras which will be equivalent, once provided a certain physical interpretation, to present the admissible potentials of the theory.

Moreover, we characterized flat connections which are known in quantum field theories to be in one to one correspondence with vacua states.

\section{Jordan algebras}

A Jordan algebra $(J, \circ)$ is a real vector space $J$ together with a bilinear product $\circ$ which is commutative and respects the following weaker version of associative property that is known as Jordan identity:

$$
a^{2} \circ(b \circ a)=\left(a^{2} \circ b\right) \circ a .
$$

We are always going to assume that every Jordan algebra has a unit (otherwise it is always possible to add it).

It is always possible to build a Jordan algebra starting from an associative, noncommutative one as follows: let $(A$,$) be an associative algebra and equip it with the anticommutator \circ: A \times A \rightarrow A$

$$
a \circ b=\frac{1}{2}(a b+b a) .
$$

It is obvious that the anticommutator is a commutative product and by direct check one can also verify that the Jordan identity holds, hence $(A, \circ)$ is a Jordan algebra. Any Jordan algebra which is isomorphic to a Jordan subalgebra of this kind is called a special Jordan algebra.

If in particular the associative algebra $A$ is endowed with an involution $*: A \rightarrow A$, that is an antilinear map $*: A \rightarrow A$ such that

$$
\begin{gathered}
\left(a^{*}\right)^{*}=a \\
(a b)^{*}=b^{*} a^{*}
\end{gathered}
$$


then the subspace $A_{s a}=\left\{a \in A \mid a^{*}=a\right\}$ of self-adjoint elements in $A$ is not a subalgebra of $(A$, but it is a Jordan subalgebra for the special Jordan algebra $(A, \circ):$ given $a$ and $b$ selfadjoint, one has:

$$
(a \circ b)^{*}=\frac{1}{2}(a b+b a)^{*}=\frac{1}{2}\left(b^{*} a^{*}+a^{*} b^{*}\right)=\frac{1}{2}\left(b a^{*}+a b\right)=a \circ b .
$$

This property can give a grasp of why Jordan algebras were initially introduced as algebraic model for quantum mechanical observables ([1],[12]): they are a natural structure if one wants to deal with products of self-adjoint operators, which are known to be physical observables in any quantum theory.

The second example that we are going to show (and which is going to play a prominent role in what follows) is the exceptional Jordan algebra $J_{3}^{8}$, whose elements are $3 \times 3$ hermitian matrices with octonion entries:

$$
J_{3}^{8}=\left\{x \in M_{3}(\mathbb{O}) \mid a=a^{*}\right\},
$$

with product which is again given by the anticommutator. It is worth to stress that $J_{3}^{8}$ is not a special Jordan algebra, due to the fact that octonions themselves form a non associative algebra (see e.g. [2]). In the following we shall omit the product and juxtaposition of two elements in a Jordan algebra will stand for their product.

We denote as

$$
[x, y, z]=(x y) z-x(y z)
$$

the associator of three elements in a Jordan algebra $J$.

The center of a Jordan algebra, denoted by $Z(J)$, is defined as the associative and commutative subalgebra of elements which associate with every pair of other elements:

$$
Z(J)=\{z \in J \mid[z, x, y]=0,[x, z, y]=0,[x, y, z]=0 \forall x, y \in J\} .
$$

This object will play many fundamental roles in the next sections.

Every time that one gives the algebraic structure of observables of a physical theroy, one has also to provide a space for physical states as representation space of said algebra. Hence we must explain what does it mean to represent a Jordan algebra.

Recall the familiar requirement of a representation $\rho: A \rightarrow \operatorname{End}(V)$ of an associative algebra $A$ on a vector space $V$ :

$$
\rho\left(a_{1}\right)\left(\rho\left(a_{2}\right) v\right)=\rho\left(a_{1} a_{2}\right) v
$$

this definition is not suitable for a Jordan algebra though. In fact it is natural to require the possibility to represent a Jordan algebra on itself, taking as representation map $\rho(a)$ the multiplication by the element $a$; but then we see that the condition above would require the algebra to be associative. We shall then take a different perspective and define a representation of a Jordan algebra as follows. Let $M$ be a vector space together with two bilinear maps

$$
\begin{array}{ll}
J \otimes M \rightarrow M & j \otimes x \mapsto j x \\
M \otimes J \rightarrow M & x \otimes j \mapsto x j
\end{array}
$$

if the direct sum $J \oplus M$, endowed with the product

$$
(j, x)\left(j^{\prime}, x^{\prime}\right)=\left(j j^{\prime}, j x^{\prime}+x^{\prime} j\right),
$$


is itself a Jordan algebra, we say that $M$ is a (bi)module on $J$ or equivalently that $J$ is represented on $M$.

\section{The exceptional quantum space}

Now we briefly resume how the structures presented in the previous section could be exploited in particle physics, for full details see [8]. The discussion that follows deals with "internal" degrees of freedom of elementary particles, thus it should not surprise that we will consider only finite dimensional Hilbert spaces and algebras.

Consider $\mathbb{C}^{3}$ as Hilbert space with standard hermitian product $\langle\cdot, \cdot\rangle: \mathbb{C}^{3} \times \mathbb{C}^{3} \rightarrow \mathbb{C}^{3}$. The vector product $\times: \mathbb{C}^{3} \times \mathbb{C}^{3} \rightarrow \mathbb{C}^{3}$ is nonassociative and $S U(3)$-invariant. Equip $\mathbb{C}$ with the trivial representation of $S U(3)$ :

$$
(g, z) \mapsto z \quad g \in S U(3), z \in \mathbb{C} .
$$

Consider $A=\mathbb{C} \oplus \mathbb{C}^{3}$ as vector space and equip it with the following $S U(3)$-invariant product:

$$
(z, Z)\left(z^{\prime}, Z^{\prime}\right)=\left(z z^{\prime}-\left\langle Z, Z^{\prime}\right\rangle, \bar{z} Z^{\prime}-z^{\prime} Z+i Z \times Z^{\prime}\right) .
$$

As matter of fact, the following theorem holds (see e.g. [15]):

Proposition 3.1. The algebra $A$ is isomorphic to the algebra of octonions $\mathbb{O}$. The subgroup of automorphisms of $\mathbb{O}$ which preserves the decomposition $\mathbb{O}=\mathbb{C} \oplus \mathbb{C}^{3}$ is isomorphic to $S U(3)$.

Now we are going to interpret $\mathbb{C}^{3}$ as the space of colour degrees of freedom of quarks and $\mathbb{C}$ as space of internal colour degrees of freedom of leptons (which is one-dimensional, meaning that leptons do not carry any colour charge, as it should be).

This interpretation connects the quark-lepton symmetry to the unimodularity condition, now we show how this binds to the existence of three generations of particles. We can arrange particles of standard model in the following table:

$$
\begin{array}{|c|c|c|c|}
\hline Q=2 / 3 \text { (Quarks) } & u & c & t \\
\hline Q=0 \text { (Leptons) } & v_{e} & v_{\mu} & v_{\tau} \\
\hline Q=-1 / 3 \text { (Quarks) } & d & s & b \\
\hline Q=-1 \text { (Leptons) } & e & \mu & \tau \\
\hline
\end{array}
$$

where $Q$ is the electric charge. This arrangement expose some sort of "triality" of the theory, meaning that each column is reapeted exactly three times, Together with the interpretation of quarklepton symmetry in terms of decomposition of octonions as explained above, this suggests the following construction.

Consider again the exceptional Jordan algebra $J_{3}^{8}$, its element are matrices whose entries are octonions which we can decompose as elements in $A$, writing:

$$
\left(\begin{array}{lll}
\xi_{1} & x_{3} & \bar{x}_{2} \\
\bar{x}_{3} & \xi_{2} & x_{1} \\
x_{2} & \bar{x}_{3} & \xi_{3}
\end{array}\right)=\left(\begin{array}{lll}
\xi_{1} & z_{3} & \bar{z}_{2} \\
\bar{z}_{3} & \xi_{2} & z_{1} \\
z_{2} & \bar{z}_{3} & \xi_{3}
\end{array}\right) \oplus\left(Z_{1}, Z_{2}, Z_{3}\right)
$$


where the diagonal elements $\xi_{i}$ 's are real numbers and, as consequence of the isomorphism above, we write every octonion $x_{i}$ as $x_{i}=\left(z_{i}, Z_{i}\right)$ with $z_{i} \in \mathbb{C}, Z_{i} \in \mathbb{C}^{3}$. Thus we obtain the following decomposition of the exceptional algebra as

$$
J_{3}^{8}=J_{3}^{2} \oplus M_{3}(\mathbb{C})
$$

where $J_{3}^{2}$ denotes the (special) Jordan algebra given by $3 \times 3$ complex hermitian matrices. Now we can look again for the automorphisms which preserve this decomposition, for which we have the following (see again [15]):

Proposition 3.2. The subgroup of automorphisms of the exceptional Jordan algebra $J_{3}^{8}$ which preserves the decomposition $J_{3}^{8}=J_{3}^{2} \oplus M_{3}(\mathbb{C})$ is isomorphic to $(S U(3) \times S U(3)) / \mathbb{Z}_{3}$.

The action of $(U, V) \in(S U(3) \times S U(3)) / \mathbb{Z}_{3}$ on an element $(H, V) \in J_{3}^{2} \oplus M_{3}(\mathbb{C})$ is given as:

$$
\begin{gathered}
H \mapsto V H V^{*} M \mapsto U M V^{*} \\
(H, M) \in J_{3}^{2} \oplus M_{3}(\mathbb{C}) .
\end{gathered}
$$

We will come back on this action very soon.

Provided a candidate for the algebra of observables, we have to select an appropriate module on which it can be represented and whose points are going to be states of our theory. For each generation of particles there are two families, that are irreducible representations of the $S U(2)$ symmetry of standard model, the most natural choice is then to consider the module $M=J_{3}^{8} \oplus J_{3}^{8}$. We do the following particle assignment:

$$
J^{u}=\left(\begin{array}{ccc}
\alpha_{1} & v_{\tau} & \bar{v}_{\mu} \\
\bar{v}_{\tau} & \alpha_{2} & v_{e} \\
v_{\mu} & \bar{v}_{e} & \alpha_{3}
\end{array}\right)+(u, c, t) \quad J^{d}=\left(\begin{array}{ccc}
\beta_{1} & \tau & \bar{\mu} \\
\bar{\tau} & \beta_{2} & e \\
\mu & \bar{e} & \beta_{3}
\end{array}\right)+(d, s, b)
$$

Looking at the expression above and to the action of $(S U(3) \times S U(3)) / \mathbb{Z}_{3}$, some considerations are mandatory:

- The diagonal elements $a_{i}$ and $b_{i}$ seems to represent new $1 / 2$ fermions whose internal space is just $\mathbb{R}$. One can think of them as Majorana spinors and there seems to be no reason to rule them out a priori from the theory, thus if one makes use of the exceptional Jordan algebra, there could be the opportunity to go slightly beyond the Standard Model.

- The action of $U \in S U(3)$ mixes different colours

- The action of $V \in S U(3)$ mixes different generetions of leptons.

We have shown how we hope to overcome the greatest flaws of noncommutative Standard model by means of modules over Jordan algebras. In order to write any significant physical action for such objects and to study some dynamics, we will start next section talking about their differential calculus, also introduced in [8]. 


\section{Connections and curvature over Jordan modules}

In the following $\operatorname{Der}(J)$ stands for derivations of the Jordan algebra, which are the linear endomorphisms of $J$ that respects the Leibniz rule for the derivation of the product:

$$
\operatorname{Der}(J)=\{X \in \operatorname{End}(J) \mid X(a b)=X(a) b+a X(b)\} .
$$

$\operatorname{Der}(J)$ is a Lie algebra with the commutator of derivations as product.

Let us denote as $\Omega^{1}(J)$ the $J$-module homomorphisms from $\operatorname{Der}(J)$ into $J$, linear with respect to $Z(J)$. We define the differential $d: J \rightarrow \Omega_{D e r}^{1}(J)$ from the relation:

$$
(d a)(X):=X(a) \quad \forall a \in J, X \in \operatorname{Der}(J) .
$$

We refer to the pair $\left(\Omega^{1}(J), d\right)$ as the (derivation based) first order differential calculus over $J$. Let $\Omega^{n}(J)$ be the $J$ module of $n$-linear antisymmetric $Z(J)$-homomorphisms from $\operatorname{Der}(J)$ into $J$, that is any $\omega \in \Omega^{n}(J)$ is a $Z(J)$ linear map:

$$
\omega: \wedge_{Z}(J)^{n} \operatorname{Der}(J) \rightarrow J
$$

then we define $\Omega(J)=\oplus_{n \geq 0} \Omega^{n}(J)$, equipped with wedge product of linear maps. We can extend $d$ to a linear endomorphism of $\Omega_{D e r}(J)$ by setting:

$$
\begin{aligned}
& (d \omega)\left(X_{0}, \ldots, X_{n}\right)=\sum_{0 \leq k \leq n}(-1)^{k} X_{k}\left(\omega\left(X_{0}, \ldots, \widehat{X}_{k}, \ldots X_{n}\right)\right)+ \\
& +\sum_{0 \leq r<s \leq n}(-1)^{r+s} \omega\left(\left[X_{r}, X_{s}\right], X_{0}, \ldots, \widehat{X}_{r}, \ldots, \widehat{X}_{s}, \ldots X_{n}\right) \quad \forall \omega \in \Omega_{n}(J) .
\end{aligned}
$$

$d$ is an antiderivation and $d^{2}=0$. We refer to $\left(\Omega_{D e r}(J), d\right)$ as the (derivation based) differential calculus over $J$.

Let now $M$ be a module over a Jordan algebra $J$. We define a connection $\nabla$ as a map from $\operatorname{Der}(J)$ into $\operatorname{End}(M)$ such that it respects the generalized Leibniz rule

$$
\nabla_{X}(a m)=X(a) m+a \nabla_{X} m \quad \forall X \in \operatorname{Der}(J), a \in J, m \in M,
$$

where we have denoted the evaluation of $\nabla$ at $X \in \operatorname{Der}(J)$ with $\nabla_{X}$. Also we require that a connection shall be linear with respect to elements in the center of the Jordan algebra:

$$
\nabla_{(z X)}=z \nabla_{X} \quad \forall z \in Z(J) .
$$

Notice that if $\nabla$ and $\nabla^{\prime}$ are two different connections, then their difference is an endomorphism of the module, in the sense that:

$$
\left(\nabla_{X}-\nabla_{X}^{\prime}\right)(a m)=a\left(\left(\nabla_{X}-\nabla_{X}^{\prime}\right)(m)\right)
$$

Thus we can classify all possible connections on a module by starting from an already known base connection and adding any module endomorphism of $M$ to it. In analogy to what we do in differential geometry, we can also define the curvature of a connection as:

$$
R(X, Y)=\left[\nabla_{X}, \nabla_{Y}\right]-\nabla_{[X, Y]} .
$$

We will say that a connection is flat if its curvature is identically zero. 


\section{Flat connections on modules of the exceptional Jordan algebra}

The classification of flat connections has huge physical relevance in quantum field theory, in particular for gauge theories. It is in fact well known that flat connections for modules over the Lie algebra of the gauge group are in one to one correspondence with the admissible ground states of any quantum field theory (see e.g. [9], [10],[11]).

For our purpose it will suffices to characterize flat connections on free modules of the exceptional Jordan algebra $J_{3}^{8}$. Dealing with free modules, the most obvious choice for a base connection $\nabla^{0}$ is the lift of the differential which is just given by differentiating component-wise elements of a module $M$. As example, on the module $M=J_{3}^{8} \otimes \mathbb{R}^{2}=J_{3}^{8} \oplus J_{3}^{8}$ one has:

$$
\nabla_{X}^{0}\left(\begin{array}{l}
x_{1} \\
x_{2}
\end{array}\right):=\left(\begin{array}{l}
d x_{1}(X) \\
d x_{2}(X)
\end{array}\right)=\left(\begin{array}{l}
X\left(x_{1}\right) \\
X\left(x_{2}\right)
\end{array}\right)
$$

And all other connections are going to be written as $\nabla=\nabla^{0}+A$ where $A$ is a module endomorphism of $M$. One can find the following result (see in [3] for theorems in the general context of free modules over any Jordan algebra):

Proposition 5.1. Let $M=J_{3}^{8}$ be a free module over the exceptional Jordan algebra $J_{3}^{8}$, every connection on $M$ is then written as:

$$
\nabla=\nabla^{0}+A
$$

where the map $A: \mathfrak{f}_{4} \rightarrow M_{n}(\mathbb{R})$ is linear.

Here the exceptional Lie algebra $\mathfrak{f}_{4}$ plays the role algebra of derivations for $J_{3}^{8}$ (see e.g. [15] for more details) and the evaluation on the derivation $X \in \mathfrak{f}_{4}$ is given by $\nabla_{X}=\nabla_{X}^{0}+A(X)$. For what concerns flat connection, one can use the characterization given above and combine it with definition (4.8) to get the following result which is quite similar to its counterpart in the setting of non abelian gauge theories:

Proposition 5.2. Flat connections on $M$ are in one to one correspondence with Lie algebra homomorphisms $A: \mathfrak{f}_{4} \rightarrow M_{n}(\mathbb{R})$. That is, for a basis $\left\{X_{\mu}\right\} \subset \mathfrak{f}_{4}$ with structure constants $\left[X_{\mu}, X_{v}\right]=$ $c_{\mu v}^{\tau} X_{\tau}$ :

$$
\left[A\left(X_{\mu}\right), A\left(X_{v}\right)\right]=c_{\mu v}^{\tau} A\left(X_{\tau}\right) .
$$

It is important to stress that, once one introduces external degrees of freedom, all of this characterization will allow us to write down the analogue of a Yang Mills lagrangian for a Jordantype quantum field theory, as we shall do in a further work.

\section{Conclusions}

We have shown how Jordan algebras could provide good ground for a rigorous mathematical formalization of Standard Model. Before doing any attempt to do some actual physics within this framework some further preparatory studies have to be conducted.

From a physical point of view one has of course to understand how to implement all gauge symmetries of Standard Model in terms of Jordan algebras; in particular the $S U(2) \times U(1)$ symmetry might be linked to the automorphisms of the special Jordan algebra $J_{2}^{4}$. 
From a more mathematical point of view, one should provide in this new language all definitions and theorems of the noncommutative formulation of Standard Model, in particular giving meaning to the notion of first order operators in a nonassociative setting.

\section{References}

[1] A.A.Albert, "On a Certain Algebra of Quantum Mechanics", Annals of Mathematics, Second Series, 35(1) $65-72(1934)$

[2] J. Baez, The octonions arXiv:math/0105155, 2002

[3] A. Carotenuto, L. Dabrowski, M. Dubois-Violette "Differential calculus on Jordan algebra and Jordan modules",arXiv:1803.08373, 2017.

[4] A.Chamseddine, A.Connes "The spectral action principle" Communic. Math. Phys. 1861997 $731-750$

[5] A. Connes, "Noncommutative geometry", Accademic Press 1994

[6] A. Connes "Noncommutative differential geometry" Pubbl. IHES 62 1986, 257 - 360

[7] A. Connes, J. Lott "Particles models and noncommutative geometry" Nucl. Phys B18 Suppl. (1990), $29-47$

[8] M. Dubois-Violette Exceptional quantum geometry and particle physics Nuclear Physics B, Volume 912, November 2016, Pages 426- 449

[9] M. Dubois-Violette (1991) Noncommutative differential geometry, quantum mechanics and gauge theory. In: Bartocci C., Bruzzo U., Cianci R. (eds) Differential Geometric Methods in Theoretical Physics. Lecture Notes in Physics, vol 375. Springer, Berlin, Heidelberg

[10] M. Dubois-Violette Lectures on graded differential geometry and noncommutative geometry in: Y. Maeda et al. "Noncommutative differential geometry and its applications to physics", Shonan, Japan (1999),pp. 245-306, Kluwer accademic pubblishers, 2001.

[11] M. Dubois-Violette, R. Kerner, J. Madore, "Gauge bosons in a non-commutative geometry", Phys. Lett. B $2171989485-488$.

[12] P. Jordan, J. von Neumann, E. Wigner, "On an Algebraic Generalization of the Quantum Mechanical Formalism", Annals of Mathematics, 1934 Princeton, 35 (1) 29 - 64

[13] K. McCrimmon "A taste of Jordan algebras" (Springer, 2004)

[14] W. van Suijlekom "Noncommutative Geometry and Particle Physics" (Springer, 2014).

[15] Ichiro Yokota Exceptional Lie Groups arXiv:0902.0431, 2009. 